\title{
Generalized Ranking for Product Aspects using Concept Hierarchies
}

\author{
K. Bharathi ${ }^{1}$, T. Venkata Satya Vivek ${ }^{2}$, G. Radha Krishna ${ }^{3}$ \\ ${ }^{1,3}$ M.Tech Scholar, Department of Computer Science \& Engineering, Grandhi Varalakshmi Venkatarao Institute of Technology, \\ Bhimavaram, India \\ ${ }^{2}$ Assistant Professor, Department of Computer Science \& Engineering, Grandhi Varalakshmi Venkatarao Institute of Technology, \\ Bhimavaram, India
}

\begin{abstract}
Now a days, the rapid growth of the internet attracts enormous customers. The need of the basic things will come to them on door to door without any risk. Users purchase the items with confidence but they should know the features of particular item. In this paper, we propose a novel framework to determine the ranking of products based on aggregation we simply use concept hierarchy to determine the products in categorical attributes with ranking taken from the consumer reviews. Basically the aspects are rated from the authenticated users who already purchased the products so that we will get the $90 \%$ genuine rating. To determine the ranking we use sentiment classifier.
\end{abstract}

Keywords: Consumer, Aspect ranking, sentiment classification, concept hierarchy review.

\section{Introduction}

E-Commerce is one of the tool for the sellers to improve their market through all over the world. Popular sites like snapdeal, flipkart, ebay and amazon attracts tremendous customers. Giant flipkart itself earns 2,846 Crores in 2014 market and it has 33000 employees working in India. These shopping sites give freedom to sell products for free with genuine authentication. Obviously 26000Million users registered with flipkart site. So to suggest the best product in all aspects we took the ratings from the customers and analyze these ratings to give some knowledge to the consumer. Even retailers also encourage to take reviews from the consumers to promote their products in all aspects. The best thing in this approach is users can give the negative feedback as their feel about the product. Generally a product can be viewed in different aspects consider the example from [1] An IPhone has more than three hundred aspects design, 3G network, panorama shoot, usability, battery, so on. Consumers can make wise purchasing decision by reading at least few reviews.

Discretization techniques are used to reduce the number of values for given continuous attributes by dividing the attribute in to range of intervals. Interval values are used to replace actual values these techniques are done based on concept hierarchies. A concept hierarchy for a given numeric attribute defines a descritization of attributes. Concept hierarchies can be used to reduce the data by collecting and replacing low level concepts by higher level concepts. Concept hierarchies can be generated using binning, histogram analysis, entropy based discretization.

\section{Problem Statement}

\subsection{System Model}

The framework contains totally four components

- Sentiment classification

- Ranking frame work
- Product aspect identification

- Concept hierarchy generation

\subsection{Working}

In this model, consumers can give their opinions regarding their products purchased in e marketing. users who are willing to purchase a product may seen different reviews. To help the consumers who are willing to buy a product we propose a novel framework for ranking the product aspects .besides that users will have flexibility to choose the products aspects in a group of classified model. In this model we explore the data by using document level sentiment classification that aims to determine a review document as expressing a positive or negative overall opinion. Another technique used was extractive review summarization which aims to summarize consumer reviews by selecting information review sentence. The algorithm works on aspect frequency count and consumer opinions over the product.

\subsection{Design Goals}

Our main goal is to make the system automation for generating useful reviews to the consumer based on the users feedback for particular product. product level aspects which are frequently occurred are considered based on product ranking identification model. Probabilistic aspect ranking algorithm

Input : Consumer review $\mathrm{R}$, Overall rating $\mathrm{O}$ and a vector of opinions $\mathrm{O}_{\mathrm{r}}$

Output: Important scores $\mathrm{w}$ for all $\mathrm{m}$ aspects. In this algorithm, it is used to identify important aspects of ranking. The aspects have the following comments. They are frequently commented in consumer reviews ii) consumer opinions has some value it. 


\section{International Journal of Science and Research (IJSR) \\ ISSN (Online): 2319-7064}

Index Copernicus Value (2013): 6.14 | Impact Factor (2014): 5.611

Sentiment classification on product aspects: The task of analyzing the sentiments expressed on aspects are called aspect level sentiment existing techniques are dependent on supervised learning. These techniques are based on training data set and labeling of training data is time consuming. A feature or aspect is an attribute or a component of an entity.

We determine whether an opinion expressed on each feature/ aspect is positive or negative.

\section{Architecture}

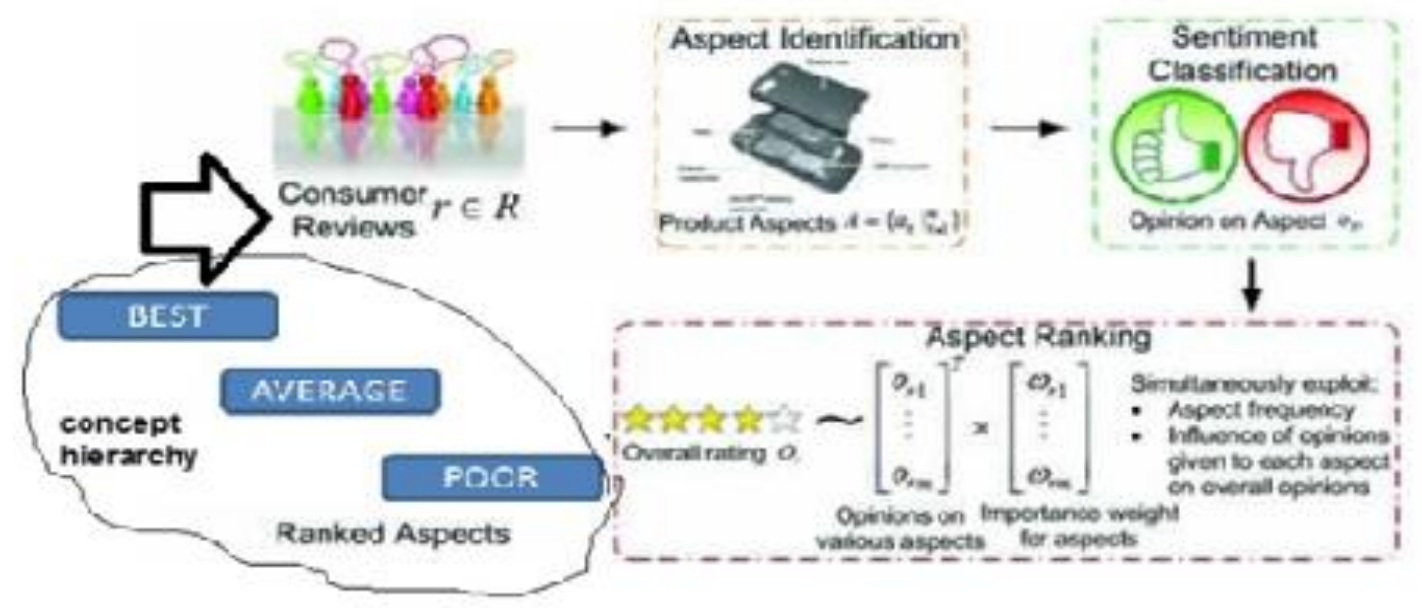

The main functionality of this approach is to extract nouns and noun phrases as candidate aspects like battery, display as nouns and good, working and average poor as noun phrases the occurrences of nouns and noun phrases are counted and frequent ones are recorded as aspects.

\section{Product Aspect Identification:}

Product aspect identification can be done using supervised or unsupervised learning techniques. Firstly extract nouns and noun phrases as aspects of a product and apply association mining to generate rules. These rules are used by consumer to make decisions.
Ranking can be done by using frequency count based method which is simply increment the counts for each word frequently occurred . Correlation based method is another technique which compares the correlation between two aspects where as hybrid method uses both the combination of frequency count and correlation.

Concept hierarchy generation: Concept hierarchies are used to map low level concepts to higher levels. In our product aspects we will concentrate on aspects at different levels based on the threshold value.

\section{Ranking Of Product Aspects:}

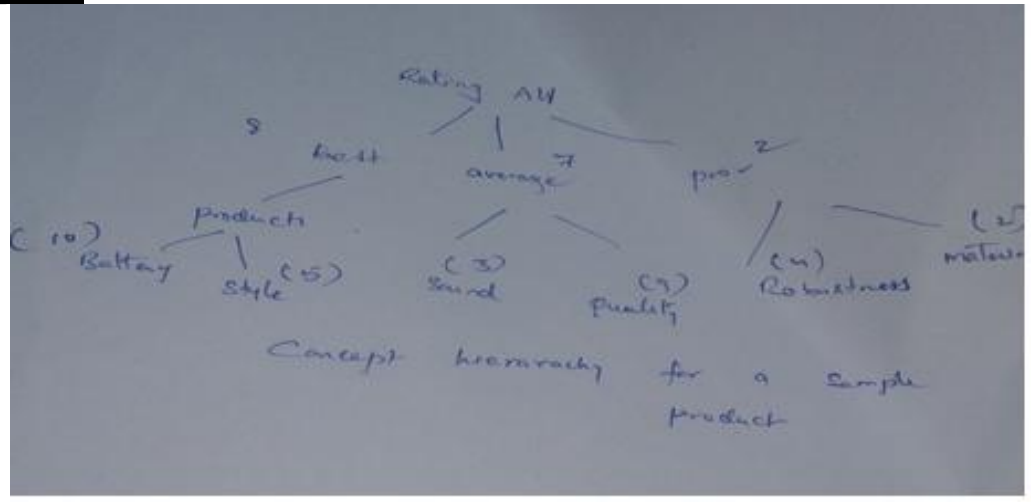

Here ranking is done using concept hierarchies. We can build tree based on frequent counts of each aspects. In this above diagram consider the low level concepts each low level cocept is assigned with a number which says the frequent occurring of the word in reviews generated by the consumers . the main advantage of this approach is we can put our own threshold value for each level to filter. The generalized case is provided with three values \{ best, average, poor $\}$.consumer can navigate through the concept hierarchies to deeper level until he finds the suitable review opinions. The leaf level contains the actual aspects of the products, users can move upward or downward to get the perfect results .Here each level is specified with the frequency count of particular aspect. In more generalized view we will get all details in a list view by travelling the leaves using 2-3 tree fashion.

$$
\text { Battery }=>\text { style }=>\text { sound }=>\text { quality }=>\text { robustness }=>
$$
material $=>$

It will arrange in L Order to navigate from one aspect to another

\section{Related Discussions}

In this paper, we mainly focus on algorithm for identifying text from the users feedback . we have two methodologies 


\section{International Journal of Science and Research (IJSR) \\ ISSN (Online): 2319-7064}

Index Copernicus Value (2013): 6.14 | Impact Factor (2014): 5.611

- Supervised

- Unsupervised

These are excellent learning techniques . supervised learning technique builds a model from the user reviews and based on the review keywords it will assign labels. It is also called sequential learning for example jin and Ho [4] learned a lexicalized HMM model to extract aspects and opinions all these methods require labeled samples where as unsupervised learning is based on characteristics features and it doesn't need any predefined working sample set . it was proposed by $\mathrm{Hu}$ and Lia [5] . They assume the product aspects are like nouns and noun phrases.

\section{Conclusion}

In this paper, we have proposed an adaptive model for consumers to make decisions easily to purchase there interested aspect of particular product. In this paper we added a new component to product aspect ranking framework called concept hierarchy. It will give the generalized view to the users Generalization is done on categorical attributes with \{Best, Average, Poor \}. Our results shown equally with the existing system with better user understandability. The document level sentiment classification is an extraordinary tool to generate frequent text patterns from the system framework.

\section{References}

[1] "Product aspect ranking and its applications " by Zheng- Jun Zha, Jinhui Tang ISSN 1041-

[2] "Multi Document summarization of evaluative text" in Proc, ACL . Sydney, NSW, Australia 2006

[3] "Estimating the helpfulness and economic impact of product reviews. Mining text and reviewer characterstics “, IEEE Trans. By A Ghose and P G Ipeirotis sept 2010

[4] "A Novel lexicalized HMM based learning frame work for web opinion mining " by W Jin and H H Ho 2009

[5] "Survey of text summarization extractive techniques " by V Gupta and GS Lehal 2010

[6] "Mining and summarizing customer reviews" M HU and B Liu, Seattle USA 2004.

[7] "A Study of information retrieval weighting schemes for sentiment analysis " by G Paltoglou and $M$ Thelwall, Sweden 2010.

[8] [8] B. Ohana and B. Tierney, "Sentiment classification of reviews using SentiWordNet," in Proc. IT\&T Conf., Dublin, Ireland, 2009.

[9] [9]B. Pang, L. Lee, and S. Vaithyanathan, "Thumbs up? Sentiment classification using machine learning techniques," in Proc. EMNLP, Philadelphia, PA, USA, 2002, pp. 79-86.

[10][10]Y. Wu, Q. Zhang, X. Huang, and L. Wu, "Phrase dependency parsing for opinion mining," in Proc. ACL, Singapore, 2009 pp. 1533-1541.

[11][11]B. Liu, M. Hu, and J. Cheng, "Opinion observer: Analyzing and comparing opinions on the web," in Proc. 14th Int. Conf. WWW, Chiba, Japan, 2005, pp. 342-351.
[12][12]A. M. Popescu and O. Etzioni, "Extracting product features and opinions from reviews," in Proc. HLT/EMNLP, Vancouver, BC, Canada, 2005, pp. 339346.

[13][13]Bing Liu" Sentiment Analysis and Subjectivity" Handbook of Natural Language Processing, Second Edition, 2010

[14][14] Aspect Ranking: Identifying Important Product Aspects from Online Consumer Reviews"by Zheng-Jun Zha, Jianxing Yu, Jinhui Tang, Meng Wang, Tat-Seng Chua Proceedings of the 49th Annual Meeting of the Association for Computational Linguistics, pages 14961505, Portland, Oregon, June 19-24, 2011.

\section{Author Profile}

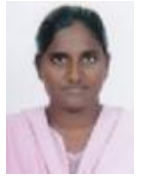

K. Bharathi, Completed her B. Tech (CSE) from Swarnandhra College of Engineering And Technology, Narasapur, India. Presently she is pursuing his M.Tech(Computer Science \& Engineering) from Grandhi Varalakshmi Venkatarao Institute of Technology, Bhimavaram, India. His Research areas include Data Mining, Cloud Security.

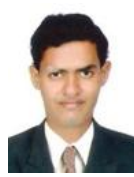

T. Venkata Satya Vivek, completed his B.Tech (CSE) from Vishnu Institute Of Technology, Bhimavaram, India and Master of Technology(Computer Networks \& Security) from KL University, Vijayawada, India. He is Pursuing his Ph.D from Dr. M. G. R Educational And Research Institute University as a Part Time Scholar. Presently he is working as an Assistant Professor (CSE Department) in Grandhi Varalakshmi Venkatarao Institute of Technology, Bhimavaram. He has published a couple of Research Papers in various International Reputed journals and Conferences. His Research Interests include Data Hiding Techniques, Cryptography, Cloud Security and Data Mining.

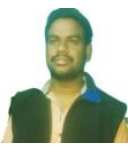

G. Radha Krishna, Completed his B.Tech(IT) from Bhimavaram Institute Of Engineering \& Technology, Bhimavaram, India. Presently he is pursuing his M.Tech(Computer Science \& Engineering) from Grandhi Varalakshmi Venkatarao Institute of Technology, Bhimavaram, India. His Research areas include Data Mining, Cloud Security. 